\title{
El Desafío de Educar a Inicios del Siglo XXI: Demandas de los Estudiantes de Ciencias Sociales a la Docencia Universitaria*
}

\author{
The challenge of Educating in the Beginning of XXI Century: Demands from Social \\ Sciences Students to University Teaching \\ O Desafio de Educar no Início do Século XXI: Demandas de Estudantes de Ciências \\ Sociais da Docência Universitária
}

\author{
Rodrigo Asún , Soledad Ruiz, , Helena Retamal, Miski Peralta, Andrea Esquivel, Luis \\ Vargas y Francisco Martínez.
}

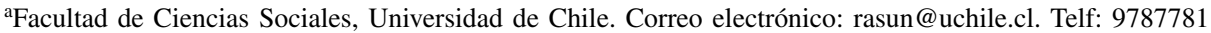

${ }^{\text {b}}$ Facultad de Ciencias Sociales, Universidad de Chile. Correo electrónico: marruiz@uchile.cl

\begin{abstract}
RESUMEN
El objetivo de este artículo es describir la forma en que los estudiantes de la Facultad de Ciencias Sociales de la Universidad de Chile conceptualizan a un "buen docente". Luego de constatar la baja producción de estudios chilenos sobre el particular, se produjeron y analizaron cualitativamente 6 grupos focales con alumnos de dicha Unidad Académica. Los resultados indican que la demanda estudiantil a sus profesores es amplia, diversa e interconectada y se encuentra centrada en aspectos diferentes al tradicional dominio de contenidos. Especial importancia tiene para los jóvenes la capacidad del profesor para ser un comunicador claro y experto, un generador de analistas autónomos, motivador, productor de evaluaciones justas, facilitador de la participación y generador de espacios de confianza. Finalmente, se discute el lugar desde el cual los estudiantes construyen sus demandas y los desafíos que estas expectativas presentan a las Universidades y al país.
\end{abstract}

Palabras clave: calidad de la docencia, educación superior, estudiantes universitarios, investigación cualitativa.

\begin{abstract}
The aim of this paper is to describe how students from the Social Sciences Faculty at Universidad de Chile conceptualize a "proper teacher". After verifying the low production of Chilean studies about the subject, six focus groups with students of this faculty were created and analysed. The results indicate that student's demands are wide, diverse, interconnected, and focused on different aspects from traditional contents expertise. For students, it is particularly important the teacher's ability to be a clear and expert communicator, a stimulus, a fair assessments producer, a facilitator of participation and a confidence spaces builder. Finally, a discussion about the position from which students establish their demands, as well as the challenges this expectations present to universities and to country are discussed.
\end{abstract}

Key words: teaching quality, higher education, university students, qualitative research.

\section{RESUMO}

Objetiva-se descrever como os estudantes da Faculdade de Ciências Sociais da Universidade do Chile conceituam um "bom professor". Após verificar a baixa produção chilena referente ao assunto, foram produzidos e analisados 6 grupos focais com alunos da referida instituição. Resultados indicam que a demanda estudantil relacionada aos docentes é

* Este artículo reporta resultados de un proyecto de investigación financiado por el programa Pulso de la Facultad de Ciencias Sociales de la Universidad de Chile y por el proyecto "Construcción y validación de un Banco de ítems para la evaluación de la calidad de la docencia por parte de estudiantes universitarios (Soc 08/06-2)”. Financiado por la Vicerrectoría de Investigación y Desarrollo de Universidad de Chile. 
ampla, diversa, interligada e encontra-se focada em aspectos diferentes do tradicional domínio do conteúdo. É particularmente importante para os estudantes a capacidade do professor para comunicar-se clara e espertamente, ser gerador de críticos autônomos, motivador, produtor de avaliações justas, facilitador da participação e gerador de espaços de confiança. Finalmente, se discute o lugar desde onde os estudantes constroem suas demandas e os desafios que essas expectativas representam às universidades e ao país.

Palavras chave: qualidade da docência, ensino superior, estudantes universitários, pesquisa qualitativa.

\section{INTRODUCCIÓN}

Desde hace algunos años, un rumor recorre el mundo docente universitario chileno: los estudiantes han cambiado y demandan un estilo de profesores distinto al que satisfacía a generaciones anteriores. De esta manera, se señala (Marín y Teruel, 2004) que anteriormente los alumnos le exigían a sus docentes principalmente dos características: a) manejar mucha información sobre el tema que trataba y b) ser un buen expositor de esa información. Sin embargo, hoy se les exigiría mucha más diversidad de habilidades: ser un buen motivador de los estudiantes, ser capaz de explicar didácticamente, ser expresivo y ameno, ser amable, ser responsable, entre otras.

Se aduce como explicación de estas transformaciones tanto el cambio tecnológico que ha modificado la relación de las personas con la información (haciéndola muy accesible), como los cambios sociales e institucionales que han facilitado el ingreso a estudios superiores a capas de jóvenes que antes, sólo como excepción, se veían en las aulas (Gallardo y Reyes, 2010).

Pese a lo extendido de este diagnóstico, no se dispone en Chile de un levantamiento sistemático de cuáles son estas nuevas demandas de los estudiantes. Como se verá más adelante, en la investigación nacional sólo es posible encontrar algunas pistas parciales respecto de estas peticiones.

Por otro lado, si bien internacionalmente la forma en que evalúan la docencia universitaria los estudiantes ha sido un tema muy estudiado desde los años sesenta, la investigación se ha concentrado sobre todo en estudios cuantitativos, siendo el cuestionario de evaluación de la docencia el instrumento de producción de información más utilizado (Cfr. Aleamoni, 1999). Estos estudios usualmente se focalizan en determinar el nivel global de satisfacción de los estudiantes, la estructura interna de dicho constructo y la validez de la medida obtenida, por lo que no siempre facilitan inferir cuáles son las demandas que les hacen los estudiantes a sus profesores.

No obstante este énfasis, de esta corriente de investigación se pueden deducir tres conclusiones que permiten comenzar a describir la demanda estudiantil actual: i) la mayor parte de los estudios empíricos constatan una estructura multidimensional en el concepto de "calidad docente", aunque con dimensiones claramente asociadas entre sí (Marsh, 1991). En otras palabras, la demanda de los estudiantes a sus profesores es temáticamente diversa, pero a la vez interrelacionada; ii) se ha encontrado en algunos estudios (Chonko et al., 2002; Francis, 2006; Okpala y Ellis, 2005; Pozo et al., 2000) que indican que lo más demandado por los estudiantes no es siempre el dominio temático del profesor, sino más bien sus habilidades pedagógicas (expositor claro, ordenado, paciente), de liderazgo (motivador, entusiasmo) y afectivas (cercanía, empatía y preocupación por el estudiante y su aprendizaje); iii) los comentarios cualitativos que los alumnos incorporan a sus respuestas a los cuestionarios se concentran en las habilidades pedagógicas del docente 
(capacidad de explicar claramente), la calidad general del curso, la organización de éste, las notas obtenidas y las habilidades interpersonales del docente (Naser-Abu y Fresko, 2009).

En relación con reportes de investigaciones cualitativas, se encontraron estudios que describen las demandas a la docencia de parte de los estudiantes, a partir de entrevistas semi estructuradas (Greimel-Fuhrmann y Geyer, 2003; Jahangiri y Mucciolo, 2008). Los resultados de estas pesquisas, en general, confirman los hallazgos cuantitativos: los estudiantes no rechazan la noción de un docente "experto" en su tema, pero agregan, otorgando similar nivel de importancia, que éste sea ordenado y claro para exponer ese contenido, que sea organizado, "comprensivo", adaptable y muestre interés por los estudiantes, que tenga un estilo de hablar atractivo, se enfoque en lo central, mantenga el orden en la clase, domine distintos métodos pedagógicos, interactúe con los estudiantes y tenga buen humor. Por el contrario, los elementos negativos de un docente serían: un estilo de exposición monótono, que no interactúe con el estudiante, que se burle de ellos, que no agregue nada a los textos y sea autoritario.

Con respecto a la producción nacional en el tema, además de reportes de resultados de evaluaciones realizadas, documentos internos de circulación restringida o el establecimiento de modelos generales de evaluación docente (Cfr. Contreras, 2010), se pueden señalar nueve estudios específicamente dedicados al tema general del proceso de evaluación docente universitario por parte de los estudiantes, todos posteriores al año 2000, lo que habla de la novedad del tópico en la comunidad científica nacional. Cuatro de estas investigaciones se ocupan de diagnosticar la percepción que tienen del proceso de evaluación docente autoridades, estudiantes y profesores (Aguilar et al., 2007; Báez et al., 2007; Salazar, 2010; Zúñiga y Jopia, 2007), mientras que dos realizan una sistematización del estado administrativo actual de la evaluación de la docencia en diversas universidades chilenas (Contreras, et. al., 2007; Salazar, 2008).

Por su parte, dos investigaciones evalúan en forma cuantitativa y global la percepción de los estudiantes respecto de la docencia universitaria. En estos estudios no se pregunta por cuáles son las demandas que los estudiantes le hacen a sus profesores, por lo que sólo es posible deducir que están más satisfechos los estudiantes de postgrado que los de pregrado (Villalobos et al., 2010) y que la satisfacción con los profesores es la variable que más explica la satisfacción general de los estudiantes con la Universidad (De la Fuente et al., 2010).

Finalmente, y siempre en el ámbito nacional, Gallardo y Reyes (2010), pese a estudiar solamente las demandas de los estudiantes respecto a la relación entre el profesor y el alumno y no en el conjunto de las conductas pedagógicas del docente, dan cuenta de la investigación más similar a la que acá se reporta.

A partir de resultados de grupos focales, estos autores señalan que los estudiantes demandan un profesor: a) cercano a ellos afectivamente, capaz de adaptar su clase a los estudiantes concretos que asisten a ella, acogiendo y creando espacios para sus opiniones y dudas, incluso personales, b) centrado en la labor docente (y no tanto en la investigación, por ejemplo), motivado por enseñar (lo que se manifiesta en su responsabilidad y en la calidad su preparación de clases) y entusiasta, c) con las habilidades necesarias para transmitir conocimientos complejos a estudiantes menos informados, d) con un manejo de grupo adecuado, que lo transforme en líder, sin ser autoritario, y capaz de exigir rendimiento con moderación y justicia, e) capaz de seducir al estudiante por las 
actividades desafiantes que plantea, por su manejo del tema, su capacidad de ejemplificar, su conocimiento del mundo profesional y/o su capacidad de relacionar los contenidos.

En contraste, los principales errores en que puede incurrir un docente son el autoritarismo (o su contrario, no ser capaz de sostener su autoridad), una actitud soberbia y lejana, el desinterés por la enseñanza, y la incapacidad de generar una clase dinámica, expresada en el hecho de utilizar una voz monocorde, falta de gestualidad y lectura textual de diapositivas.

Pese al interés de esta última publicación, se confirma que el campo de conocimiento de las demandas y evaluación de la docencia universitaria por parte de los estudiantes en Chile aún requiere ser construido. El propósito de este artículo es contribuir a llenar, en parte, este vacío a través de la descripción y comprensión de las demandas que los actuales estudiantes universitarios le hacen a sus docentes. Específicamente, los resultados dan cuenta de las características que constituyen a un "buen docente" para los estudiantes, cómo las relacionan entre sí y qué orden de prioridad les asignan. Con ello, se espera realizar un aporte sobrio, aunque relevante, al debate acerca de la calidad de la educación superior en Chile.

\section{METODOLOGÍA}

La investigación se realizó entre 2008 y 2009, con estudiantes de la Facultad de Ciencias Sociales de la Universidad de Chile. Esta Unidad académica dicta actualmente 4 carreras de pregrado: psicología, sociología, antropología y educación básica e inicial.

Para la realización de este estudio se utilizó un enfoque cualitativo, en la medida en que permite acceder a los contenidos y valoraciones que los sujetos despliegan en sus discursos respecto a los temas investigados. La técnica de producción de datos fue el grupo focal. Se utilizó esta modalidad pues permite indagar en profundidad las temáticas que se plantean, favoreciendo que se produzcan intercambios personales y discusiones sobre sentimientos, actitudes, creencias, experiencias y comportamientos (Morgan, 1990).

Se utilizó muestreo cualitativo con definición a priori de la muestra (Flick, 2002). Esto quiere decir que los criterios muestrales fueron previamente definidos a la luz de los antecedentes empíricos preexistentes. Es decir, se escogieron criterios que parecieron relevantes para dar cuenta de las variaciones discursivas de los estudiantes, a saber: carrera, año de estudio y sexo de los alumnos. De esta forma, se programó la realización de cuatro grupos focales mixtos y con participación de estudiantes de las cuatro carreras de la Facultad, pero homogéneos en su curso, dando como resultado dos grupos focales de estudiantes de los primeros dos años y dos de los terceros y cuartos años. A lo anterior se agregó 2 grupos más para compensar algunos problemas emergentes de representación: uno integrado solamente por alumnas de la carrera de educación básica e inicial y otro por estudiantes de la especialidad de antropología física, perteneciente a la carrera de antropología.

La selección de los participantes de cada grupo focal fue realizada por miembros del equipo de investigación, quienes reclutaron a los participantes bajo la promesa de que lo dicho por ellos en este espacio sería anónimo y utilizado solamente para mejorar 
la docencia de la Facultad y los mecanismos de evaluación de la misma. Los grupos fueron moderados por miembros del equipo de investigación distintos a quienes los habían producido.

El análisis de los datos se realizó según los procedimientos estipulados por la "grounded theory" (Glaser y Strauss, 1967; Strauss y Corbin, 1990). Para tal efecto, el primer paso fue la codificación de los datos obtenidos, que significó identificar -a través de lectura y discusión grupal- los fenómenos de interés presentes en los diferentes pasajes o extractos del material transcrito y registrarlos verbalmente a través de su etiquetamiento.

Los resultados generados a partir de estas categorizaciones se fueron integrando en unidades mayores a medida que progresaba la investigación, creando una clasificación jerárquica que permitió organizar y establecer la prioridad de las demandas de los estudiantes. Con ello se obtuvieron los resultados que se exponen en el apartado siguiente.

\section{RESULTADOS}

Se exponen las demandas de los estudiantes en cuatro apartados: en el primero se examinan las características que los alumnos consideran mínimas para el ejercicio de la docencia, pero que no son suficientes para calificar como "bueno" a un profesor. En las siguientes tres secciones se presentan las propiedades que distinguen a los buenos docentes de aquellos que sólo cumplen con los requisitos mínimos.

En relación con cada una de estas temáticas, se distinguen las peticiones de los estudiantes en dos categorías que han emergido desde el análisis: a) demandas unidireccionales, es decir, dimensiones en que se pide más o menos de una característica y; b) demandas de justo medio, en que si bien se pide más (o menos) de alguna propiedad, se considera igualmente inadecuado o incluso peor excederse en ese comportamiento. Dicho en palabras de una estudiante, frente a algunos temas hay una "delgada línea" en que deben intentar situarse los docentes.

\subsection{CONDICIONES MÍNIMAS PARA SER BUEN DOCENTE}

DOMINIO DEL TEMA

Los estudiantes en general consideran que una característica básica de cualquier docente consiste en tener dominio de los temas que pretende enseñar. De hecho, ello debiese ser tan evidente que no tendría que haber nadie que haga clases en la Universidad de Chile que no domine los temas sobre los que enseña:

"Que los docentes tienen que tener dominio de sus cátedras debería ser algo imprescindible, o sea no debería ni siquiera cuestionarse, no deberíamos tenerlo en consideración para decir: ese es un buen profe" (exclamaciones de apoyo por parte del grupo).

Para los estudiantes, indicadores de que se está en presencia de un docente con dominio de su tema son: a) que conozca información actualizada sobre el particular, es decir, que esté enterado de los últimos avances de la investigación o reflexión sobre el tema; b) que sea capaz de responder, sin demasiadas dudas, a las preguntas de los estudiantes; c) que enseñe tanto técnicas aplicadas como sus sentidos más profundos; y d) y que sea capaz de recomendar bibliografía complementaria pertinente si un estudiante lo solicita. 
Para los estudiantes, la responsabilidad del profesor es un indicador del compromiso que éste tiene con la docencia y, en esa medida, del respeto que demuestra en los hechos por sus alumnos. De igual forma, se considera una injusticia que el docente exija responsabilidad a sus alumnos sin ser el mismo responsable con sus tareas.

Los principales elementos que constituyen indicadores de la responsabilidad de un profesor son: a) realizar todas sus clases o avisar con tiempo si no se va a acudir; b) llegar y terminar las clases a la hora y; c) entregar a tiempo las evaluaciones. Este último punto es considerado crucial pues, además de dar información oportuna al estudiante sobre su rendimiento, constituye para ellos una retroalimentación al aprendizaje.

No obstante, es importante recalcar que la responsabilidad no es un indicador suficiente para calificar a un docente como bueno. Al contrario, si el docente sólo cumple sus compromisos sin tener otras propiedades positivas, puede ser acusado de cumplir sólo formalmente con su tarea.

No obstante lo anterior, si bien es cierto que el discurso general señala que la irresponsabilidad, aún en profesores connotados, implica ser un mal docente, hay algunas voces que se abren a la posibilidad de excepciones:

"Que el profe llegue 5 minutos tarde o 10 minutos tarde, o que entregue las pruebas con dos semanas de atraso, da lo mismo si es muy bueno. Como que siento que un buen profesor se desliga un poco de esas cosas pequeñas".

\subsection{CARACTERÍSTICAS QUE DISTINGUEN AL BUEN DOCENTE:} COMPORTAMIENTO DOCENTE EN EL AULA

\section{COMUNICADOR CLARO}

Existe acuerdo entre los estudiantes respecto de que una característica del buen docente es la capacidad de exponer en forma clara el conocimiento que quiere transmitir. No obstante, hay importantes diferencias en el énfasis con que ella es señalada en los grupos focales: no es demasiado acentuada en el discurso de los estudiantes de cursos superiores, pero es considerada básica y fundamental por los estudiantes de primeros años, posiblemente porque éstos se están iniciando en disciplinas de las que no tienen referencias previas.

Los principales indicadores que los alumnos distinguen para evaluar la capacidad explicativa de un docente son: a) esquematizar, ordenar y secuenciar los contenidos de manera de hacerlos más comprensibles, b) trasmitir a los alumnos una idea global del curso y su sentido en el marco de la carrera, c) hablar mirando a los estudiantes y no a la pizarra, con un ritmo, dicción y volumen adecuado, y, d) graduar la complejidad de las materias a los conocimientos previos de los estudiantes, sin ser muy simple, pero tampoco incomprensible.

Los principales problemas que puede tener un docente en este punto son el desorden expositivo y enfocarse sólo en una parte muy especializada de los contenidos, de manera que el estudiante no comprenda el marco general en que se encuentra ese discurso. En este sentido, la imagen más recurrente de un mal docente se traduce en la de un profesor "divagador", que se concentra sólo en una parte de los contenidos del curso (usualmente 
aquellos que son más relevantes para el académico, por su propia investigación o especialización laboral):

"Lo otro que encuentro fundamental es que sean estructurados, que un buen docente tiene que estructurar sus clases y no puede por el hecho de que sea un, no sé, un doctor en el asunto, un divo, llegar y tirarse el rollo y decir siempre lo mismo, siempre lo mismo".

\section{COMUNICADOR EXPERTO}

Los estudiantes, y muy especialmente los de tercer y cuarto año valoran, más que la claridad expositiva, a los docentes que se comportan como expertos a la hora de comunicar y transmitir los contenidos de sus asignaturas. Por comunicador experto se entiende que los académicos: a) sean capaces de extractar lo esencial de la información que entregan sin perderse en detalles, b) utilicen ejemplos de la realidad contingente a la hora de explicar las temáticas, c) empleen su experiencia laboral o como investigador para ejemplificar sus materias y d) realicen el ejercicio de ligar sus contenidos con otros temas o con conceptos provenientes de áreas distintas:

"O ligar las cosas con experiencias, esa cuestión yo creo que es súper motivante, lo que yo me acuerdo de profes como que siempre te están contando historias, que se nota que lo que te están enseñando ellos como que lo han vivido, no es que se lo sepan por libro no más [...]. Yo me acuerdo (...) que el profe nos contaba de, qué se yo, los pescadores artesanales [....... son esas cosas que tú te acuerdas después y como que lo puedes relacionar con cosas, puedes aplicarlo después. Yo creo que son esas las cosas que te van interesando porque de repente tenis cosas tan duras y tan, y tú dices y dónde lo aplico y como que... no sé, eso más que nada, la cuestión de la experiencia yo creo, que se note que el tipo sabe de verdad."

En este ámbito se debe tener en cuenta que uno de los principales problemas que puede experimentar el profesor es ser visto como reproductor de un discurso que los estudiantes pueden encontrar, casi en su totalidad, en otros dispositivos pedagógicos (como libros, web, presentaciones).

Lo que se le solicita a un docente en este punto es que sea capaz de producir un discurso significativo, tanto por la selección que ha realizado de los contenidos, como por su capacidad de situarlo en el marco de otros contenidos teóricos y prácticos. Especial relevancia tiene, para evaluar bien a un profesor en esta área, que sea capaz de ligar la teoría con la práctica, a través de la aplicación de la primera a la coyuntura actual, al mundo laboral, a la comprensión de otros temas o a la interpretación de sus propias investigaciones o experiencias.

No obstante, lo anterior debe hacerlo sin caer en la reiteración o autorreferencia excesiva. Por ello, esta demanda constituye la primera de las "delgadas líneas" en las que el docente debe moverse. Así, un académico que incorpore en la medida adecuada esta experiencia será muy valorado y facilitará que los estudiantes sientan que adquieren conocimientos significativos. Sin embargo, si exagera la medida (ya sea por excesivas repeticiones o por relatar experiencias muy personales), puede ser considerado un profesor "divagativo". 
Además de lo anterior, un buen docente debe ser capaz de utilizar su cátedra no sólo para entregar contenidos, sino para generar motivación por el aprendizaje. En este sentido, los buenos docentes son quienes son capaces de interesar en tal medida a sus alumnos que los comentarios y reflexiones sobre los contenidos presentados, se extienden fuera de la sala de clases. El nivel máximo de esta capacidad se logra si el estudiante se ve motivado a seguir aprendiendo por su cuenta, buscando bibliografía e investigando.

Las formas en que el docente puede generar esta motivación y seducción son variables, pero todas ellas tienen dos denominadores comunes: a) el docente debe expresar entusiasmo y pasión por el tema que está tratando, desarrollando un discurso convincente sobre su relevancia y exponiendo que lo que enseña está enraizado en su propia biografía académica (a través de sus investigaciones y/o actividad laboral) y; b) debe expresar placer por la tarea pedagógica, demostrando que le gusta e importa formar alumnos.

Además, un docente motivador es capaz de conducir una clase: a) dinámica: es decir, una en que la exposición es lo suficientemente activa como para mantener la atención (por la vía de un buen uso del espacio de la sala, un ritmo adecuado y cambios en los énfasis); b) participativa: en el sentido de facilitar que los estudiantes intervengan dando sus impresiones respecto a lo que están aprendiendo y; c) provocadora: lo que implica que el docente debe plantear preguntas respecto al tema para que los estudiantes se queden reflexionando:

"Como que hay profes que saben mantenerte como intrigado, así como saber que no te pueden decir todo, como para que tu quieras estar en su clase, como que te va contando un cuento que te entretiene (...) los que son capaces en el fondo, claro, de ir ligando lo que están enseñando con la experiencia, que te vayan haciendo pensar".

Al contrario, en el campo motivacional, lo peor que puede expresar un profesor es desinterés por dictar clases:

"Como que lleguen a hacerte un favor a clases, como si te estuviera haciendo un favor por enseñarte".

Toda esta descripción refleja que, en los discursos de los estudiantes, la responsabilidad principal y casi exclusiva por la calidad de la docencia y la motivación de los alumnos es del profesor. Las afirmaciones sobre su propio rol en el proceso y autocríticas respecto de su comportamiento son escasas. Por ello, si bien se reconoce que algunas veces la retroalimentación que dan a los profesores sobre como están recibiendo la docencia es poco clara, se describe esta situación como una característica intrínseca de los alumnos, no como un problema a solucionar.

\section{CREADOR DE ESPACIOS DE PARTICIPACIÓN}

Los estudiantes expresaron vivir con emociones muy intensas su proceso de aprendizaje y, al situarse como aprendices de un tema, declararon sentirse muy expuestos a la descalificación por ignorancia, por lo que manifestaron ciertas dificultades para participar dentro de las clases. En virtud de lo anterior, una de las características más celebradas de un buen docente es ser capaz de generar un espacio seguro (no expuesto al ridículo) en que los estudiantes puedan aportar sus dudas, visiones y propuestas. 
Esta demanda por espacios de participación tiene importantes matices según el curso de los estudiantes. Así, en los alumnos de primer y segundo año, la petición suele estar más vinculada a poder tomar la palabra para preguntar o aplicar un contenido y utilizar la retroalimentación docente para evaluar si es que han comprendido adecuadamente:

"Porque uno llega a un choque en que no entiende, no sabe funcionar, no sabe cómo interactuar y se vuelve algo poco retroalimentativo, y al final uno duda realmente qué es lo que aprendió y si lo aprendió bien".

Por su parte, los estudiantes de tercer y cuarto año consideran que ellos ya están en condiciones de aportar algunos contenidos al conocimiento de lo tratado. Por ello, su demanda está más relacionada con que el docente brinde espacio para acoger sus aportes y sea capaz de recoger sus comentarios e integrarlos, haciendo del aula un lugar de discusión donde profesor y alumnado experimenten un aprendizaje conjunto.

"Lo que les falta a gran parte de los profesores de acá es escuchar a sus alumnos y que haya una retroalimentación fuerte entre alumno y profesor".

Por lo tanto, lo que se pide es que los docentes en su clase: a) creen un espacio de confianza que permita las preguntas e intervenciones; b) respondan y retroalimenten las consultas de sus estudiantes, sin burlarse explícita o implícitamente de los errores cometidos; c) planteen preguntas o debates, cediendo luego la palabra y la reflexión; d) estén dispuestos a una discusión no descalificatoria con sus estudiantes, si alguno de éstos tiene una opinión distinta; e) estén abiertos a incorporar y aprender de las reflexiones de los alumnos.

No obstante, este énfasis en la necesidad de que el profesor brinde espacios de participación no implica que los estudiantes prefieran sin reservas los cursos en que el profesor co-construye el contenido del curso con los educandos. Por el contrario, en general, se demanda que el profesor no abdique de su rol de conductor del proceso de enseñanza. Generalmente, eso implica que el profesor mantenga, cuando menos, una parte expositiva en el curso y, por lo tanto, son vistas con bastante sospecha aquellas asignaturas en que el docente descarga mucho del peso del proceso de aprendizaje en los alumnos.

Lo anterior implica que mucha o poca participación puede ser igualmente negativa. Un buen docente es quien logra el equilibrio entre ambos extremos: esta es la segunda "delgada línea" en que deben moverse los académicos.

\section{FORMADOR DE ANALISTAS AUTÓNOMOS}

Muy relacionada con la destreza que se ha denominado "comunicador experto", se encuentra otra de las habilidades de un buen docente: la capacidad de brindar herramientas para que el estudiante pueda, en forma autónoma, comprender más profundamente los contenidos aprendidos en clase e interpretar más claramente la realidad que le rodea. En palabras de los estudiantes, uno de los principales deberes de los docentes es "potenciarlos".

Según el discurso de los estudiantes, se espera de un buen docente que entregue "tips" que permitan esta comprensión superior. Estos "tips" consisten en: a) herramientas conceptuales; b) herramientas metodológicas; c) ejemplos de la forma en que se pueden conectar entre si los distintos conceptos y teorías y; d) ejemplos de aplicación de dichos conceptos a situaciones concretas. 
Esta petición se liga con que se entiende que el principal objetivo de un estudiante y su principal placer es ser capaz de comprender la realidad que los rodea y les es significativa. La demanda tácita a los docentes es que sean capaces de enlazar con sus intereses, lo que usualmente tiene que ver con presentar temas más cercanos a su vida cotidiana, de coyuntura de la sociedad en que viven o asociados con la capacidad de desarrollar una mirada "crítica" o novedosa respecto de la misma.

"Me pasa a veces que no sé, hablan y yo después llego a mi casa a hablar y nadie me pesca (risas), le dices como a tu polola, a tu hermano, como "ohhh, hoy día en clases..." y te cambian de tema, no te pescan. Cuando pasa eso es cuando el profesor da como justo en lo que uno estaba buscando".

Frente a esta buena docencia, el estudiante tiene una serie de reacciones que se entienden como una retribución simbólica para el profesor: se asiste más a sus clases, se estudia con mayor intensidad, se leen los materiales entregados, se es más responsable en las actividades y plazos, entre otras conductas. Ello se explica porque, si bien aprender es principalmente caracterizado como una actividad placentera, también se tiene claro que implica un sacrificio que tiene alternativas muy competitivas, como la sociabilidad con sus compañeros, descansar, asistir a otras actividades, etc. Por ello, si el docente se ha esforzado y demostrado compromiso con enseñar, el estudiante puede responder a ese compromiso perseverando en aprender.

Por otro lado, si bien los alumnos demandan una clara relación entre los contenidos que les enseñan y la "coyuntura", la investigación o el medio laboral, usualmente limitan esa petición al proceso de enseñanza - aprendizaje que ocurre en el aula, sin dar demasiada importancia a las labores de investigación y extensión de la Facultad. De esta forma, no construyen ninguna petición relacionada con una mayor participación en investigaciones o actividades de extensión como parte del proceso formativo. Si el profesor está en contacto con el medio social externo, ello sólo es valorado en la medida en que posibilita la transmisión de su experiencia y conocimiento del mundo laboral a los educandos, específicamente en la sala de clases.

De la misma manera, la investigación es principalmente percibida como una actividad que compite con la dedicación de los académicos a la docencia, por lo que puede ocurrir que existan algunos académicos que dejen de lado a sus estudiantes por sus investigaciones:

"Hay profesores que se les nota mucho que los trabajos que nos hacen son para aportar para sus investigaciones, que es un tema que se la rebusca y no está preocupado de que nosotros aprendamos y que seamos profesionales en el futuro comprometidos con la realidad social del país".

En otras palabras, los estudiantes sostienen que lo que da sentido a la existencia de los académicos es su rol de docentes, por lo que es importante que éstos sepan que hacerles buenas clases es su principal deber. Por ello, cuando se encuentran con lo que definen como "malos profesores", experimentan intensos sentimientos negativos expresados en frases como "estar siendo objeto de una burla", "sentir frustración", "estar en una situación horrible", entre otras expresiones.

En este marco, los alumnos son capaces de construir sólo dos explicaciones de esta "mala docencia". En primer lugar, algunos creen que, ya que ser buen profesor no es difícil, el problema debe estar en la voluntad: no hay real compromiso con la enseñanza, pues se tiene predilección por otras actividades o simplemente no se está interesado en enseñar. 
"Yo creo que no hay que ser tan seco como para preparar una clase, es cosa de saber, o sea, de leerse los textos, saber de qué estás hablando, pero hacer un breve resumen y después empezar como la cosa práctica, aplicarlo, a que se genere como un conocimiento como de verdad de los textos".

La segunda explicación que se construye es más estructural: se señala que para llegar a ser buen profesor hay que disponer de cierto grado de capacitación en pedagogía o de experiencia docente, que muchos académicos no han recibido, por lo que la Universidad debería preocuparse de formarlos antes de darles la responsabilidad de conducir un curso.

\subsection{CARACTERÍSTICAS QUE DISTINGUEN AL BUEN DOCENTE: COMPORTAMIENTO INTERPERSONAL}

\section{CREADOR DE RELACIONES DE RESPETO, APOYO Y EMPATÍA CON LOS ESTUDIANTES}

Una de las principales demandas de los estudiantes es que los profesores tengan buenas relaciones con ellos. Dada la asimetría de conocimiento y poder entre ambos estamentos, los alumnos consideran crucial para su aprendizaje una buena relación con los académicos.

¿En que consisten esas buenas relaciones? Algunos elementos ya señalados se sistematizan en esta sección: a) mantener una relación de respeto, no burlándose de la falta de conocimientos de los estudiantes, ni tratándolos en forma paternalista, como si esta ignorancia implicara bajas capacidades de aprender; b) estar disponible a dialogar, aceptando comentarios y aportes a la clase; c) estar disponible fuera y dentro de la clase para responder consultas (por medios presenciales o no presenciales); d) empatizar con los intereses de los alumnos, detectando cómo están recibiendo la docencia y; e) flexibilizando los contenidos de los cursos para acercarse a ellos.

Subyace a esta demanda el hecho de que los estudiantes evalúan que el aprendizaje que ellos están realizando no es emocionalmente sencillo ni cognitivamente fácil, por lo que requieren a un profesor que los "apoye" en el proceso.

"Es importante que los profesores fuera del horario de clases tengan tiempo para uno y para sus dudas, de repente son dudas muy tontas, o te ayudan a solucionar cosas a futuro para uno, pero de repente es importante sentir como ese apoyo de parte de los profesores porque es lo único que uno tiene cuando se está formando en algo que nunca ha visto antes entonces... poder ir a hacerle consultas y que esté todo el día y que uno los pueda molestar, que no te cierre la puerta en la cara o te diga no, no, no puedo".

Por otro lado, los principales miedos que los estudiantes enfrentan al entrar en una interacción más activa con un docente, consisten en que el profesor utilice su posición superior en jerarquía y conocimiento para hacer mofa de su falta de dominio (hacerlos "sentirse tontos" o "humillados por su ignorancia" son las formas habituales de expresar este temor). Estas expresiones son, a veces, utilizadas para explicar por qué tienen comportamientos pasivos dentro del aula.

No obstante lo anterior, si bien no se considera legítimo que el docente actúe despóticamente, igualmente se le pide que sea capaz de mantener el respeto del curso. Se encuentra acá la tercera de las delgadas líneas de nuestro análisis: el profesor no debe caer en el autoritarismo, pero tampoco debe abandonar su rol directivo: 
"Tiene que ser el manejo del grupo y que cuando llegan como que nos comemos al profesor en el fondo, porque uno le habla y el profesor como que se asusta".

\section{PRODUCTOR DE COORDINACIÓN Y DIÁLOGO CON OTROS DOCENTES}

Si bien esta dimensión de las demandas de relaciones interpersonales no es tan recurrente, también es posible señalar que se pide a los buenos educadores ser capaces de generar relaciones de cooperación y discusión a nivel académico con otros docentes (especialmente con aquellos pertenecientes a distintas escuelas o líneas de desarrollo). En este sentido, un buen profesor será capaz de coordinar los contenidos de su cátedra con las del resto de la carrera y, además, mantendrá relaciones de debate en espacios académicos con sus colegas (participando en seminarios, por ejemplo).

Al contrario, un mal docente no se preocupará más que de sus propios contenidos y no dialogará con sus colegas, utilizando a los estudiantes para triangular con ellos sus opiniones sobre el resto de los académicos:

"Sería interesante que todo lo que digan lo digan esperando una respuesta del otro y que el otro pueda defenderse, porque si no caemos en el pelambre del otro, porque son adultos y más encima académicos".

\subsection{CARACTERÍSTICAS QUE DISTINGUEN AL BUEN DOCENTE: ORGANIZACIÓN Y EVALUACIÓN} DE LOS CURSOS

\section{PRODUCTOR DE EVALUACIONES PERTINENTES Y JUSTAS}

Los estudiantes consideran que las evaluaciones y su validez son un componente muy relevante de la calidad del docente. En ese sentido, lo que le piden a una buena evaluación es que sea: a) coherente con la dificultad y tipo de destrezas desarrolladas en clase, de manera que no se soliciten habilidades que no se hayan formado previamente; b) justa, en el sentido de reflejar lo que ellos perciben es su nivel real de aprendizaje y esfuerzo; c) formalmente acotada, lo que significa que no se le pida al estudiante repetir casi la totalidad de los contenidos en largas horas de escritura, y; d) abierta, de modo que el alumno pueda presentar su propia perspectiva de los conocimientos adquiridos. Los estudiantes perciben las evaluaciones como espacios para expresarse, no solo como instrumentos de evaluación.

Además, en relación con la corrección, se considera que las evaluaciones deben estar fundamentadas (se menciona la utilidad de las pautas de evaluación), se deben entregar los resultados oportunamente y que se debe generar un espacio para la revisión conjunta de los errores cometidos:

"Claro, que te retroalimenten respecto a la evaluación, ese en el fondo es el sentido que tiene... bueno también claro, tiene todo el sentido desde el punto de vista de discriminar a los mejores estudiantes, qué se yo pero, pero en términos de aprendizaje yo creo que es un proceso súper necesario, que muchos profesores no están acostumbrados a hacer".

Desde el punto de vista de las evaluaciones, se consideran negativas tanto las pruebas muy difíciles que asignan malas calificaciones a muchos, como aquellas muy fáciles en que todos obtienen buenas calificaciones. Las primeras son leídas como manifestaciones de un profesor autocentrado (“divo"), que no tiene en cuenta que los estudiantes tienen otras obligaciones, mientras que las segundas dan la impresión de que el profesor tiene 
poco interés en que los estudiantes aprendan o utiliza las notas como un virtual soborno para que ellos no se quejen de la calidad del curso.

Constituye esta la cuarta de las "delgadas líneas" en que se debe mover un educador universitario: se le demanda producir una evaluación que, desde la perspectiva de los estudiantes, sea desafiante, pero sin que sea muy difícil de resolver.

\section{PLANIFICADOR DOCENTE EFICAZ}

Pese a no ser un elemento tan acentuado como otros, los estudiantes también consideran que es característico de un buen docente que éste sea capaz de: a) planificar y actualizar continuamente sus clases; b) organizar su curso cumpliendo con la totalidad de los contenidos del programa y; c) estructurar las clases de manera que exista coherencia entre los objetivos de aprendizaje a lograr y las metodologías a emplear.

En relación con el primer punto, se considera que es indicador de un buen docente que éste no replique una y otra vez las mismas clases y ejemplos. De esta manera, se optimiza la posibilidad de que la cátedra tenga sintonía con la coyuntura y se demuestra el compromiso del académico. Respecto al cumplimiento del programa, se valora que un profesor sea capaz de cubrir todas las unidades planificadas, en tiempos adecuados (por ejemplo, sin acelerar el ritmo a último momento) y con evaluaciones pertinentes y espaciadas. No parecen adecuados los docentes que "se quedan pegados" en alguna unidad.

No obstante lo anterior, esa meta debe ser matizada con la demanda de que el docente debe disponer de la suficiente empatía y disposición como para cambiar su programa en función de las solicitudes y motivaciones de los alumnos. Se observa acá una quinta delgada línea en los requerimientos al académico: debe ser capaz de planificar un programa y abordarlo en su totalidad, pero también tiene que estar disponible para su modificación, al menos, parcial:

"O sea la capacidad de empatía del profe para mi va en que tu puedes tener tu programa muy bien programado, pero que si las cosas se tienen que modificar porque la estructura del curso, porque la gente del curso o porque las cosas no salieron nomás, ser capaz de modificarlo".

En relación con la demanda por coherencia entre la metodología de enseñanza y los objetivos pedagógicos del curso, los alumnos consideran que existen ciertos contenidos para los cuales resulta más pertinente usar metodologías participativas y otros que se ajustan mejor a clases de carácter expositivo, siendo un buen profesor el que mejor modula los métodos a emplear según las temáticas y sus destrezas personales.

\section{UTILIZADOR EFICAZ DE RECURSOS COMPLEMENTARIOS}

Un último elemento valorado por los alumnos (aunque no demasiado señalado) es que los cursos cuenten con recursos que apoyen efectivamente la actividad pedagógica.

Un tipo de recurso complementario, especialmente señalado por estudiantes de los primeros años, es la bibliografía del curso. Se espera que ella sea un soporte de la cátedra, esté en concordancia con sus objetivos (y no distraiga de éstos), sea actualizada y posea una dificultad adecuada para su nivel.

Por otro lado, si bien no hay una fuerte demanda por el uso de nuevas tecnologías, se señala que el uso de proyectores de imágenes es visto como una oportunidad, por la posibilidad que brindan de ver ejemplos prácticos, acceder a material de Internet, visualizar 
imágenes o películas, entre otras alternativas. No obstante, es criticado el mal uso que algunos docentes le dan, pues al transcribir totalmente su clase a esa herramienta y dedicarse a leerla, los profesores hacen muy rígida la secuencia de contenidos y bloquean la interacción con los estudiantes ("apagando el conocimiento", al decir de un estudiante), sin aprovechar las ventajas de esa tecnología.

\section{CONCLUSIONES}

Lo primero que se puede señalar respecto a la demanda de los estudiantes de la Facultad de Ciencias Sociales de la Universidad de Chile hacia sus profesores es que, si bien comparte la mayor parte de los elementos señalados en las investigaciones preexistentes reportadas en la presentación, tiene algunas especificidades que se hacen más evidentes en la comparación con las investigaciones internacionales.

Así, es posible notar que el discurso descrito en estos últimos estudios tiene más parentesco con las demandas de los estudiantes de primero y segundo año, que con la de los estudiantes de cursos más avanzados, ya que está mucho más centrada en aspectos expositivos (claridad, orden de las presentaciones, ritmo adecuado de habla) y enfatiza menos la capacidad del docente de generar pensamiento autónomo o ser un "comunicador experto" capaz de relacionar su materia con la práctica, el mundo laboral u otros contenidos.

Por otro lado, el estudio aquí reportado tiene muchas más afinidades con los resultados de la investigación nacional de Gallardo y Reyes (2010). En ambos casos, se demanda un comunicador claro, afectivamente cercano y respetuoso, justo, pero exigente, capaz de motivar, centrado en la docencia de pregrado y "experto", capaz de encantar con su dominio a los estudiantes. La diferencia parece estar en que los estudiantes de la Facultad de Ciencias Sociales demandan menos un docente cercano afectivamente y más un "experto" capaz de formar analistas autónomos. Así, se pide de los profesores respeto, preocupación y apoyo en el aprendizaje, pero no aparece una demanda explícita por un conocimiento personal entre alumnos y profesores o que el docente sea capaz de percibir y acoger los estados de ánimo de sus alumnos.

Lo segundo que se puede constatar es la intensidad emocional y relevancia que tiene la calidad de la docencia para los estudiantes, lo que se denota, tanto en la forma en que hablan del tema, como en la centralidad que asignan a la formación de pregrado en las funciones de la Facultad: es posible afirmar que, para los estudiantes, el centro de su actividad debería estar situada en el proceso de enseñanza-aprendizaje que se produce en el aula. Consecuentemente, los académicos son percibidos y evaluados esencialmente en el ejercicio de esa labor, siento el resto de sus funciones casi invisibles desde la perspectiva de los estudiantes.

En tercer lugar, se puede apreciar la complejidad de la demanda que les hacen los estudiantes a sus docentes. Como se observa en la Figura 1, se les pide a los docentes doce habilidades distintas, dos de carácter mínimo, pero diez más elaboradas, que pueden ser agrupadas en tres campos separados: comportamiento en el aula, relaciones interpersonales y organización y evaluación de los cursos.

Si bien no todas las demandas parecen tener la misma relevancia, pues son señaladas con diferente énfasis y frecuencia en los grupos focales, al menos 7 de ellas (para las 
que se ha utilizado letra de mayor tamaño) podrían ser consideradas muy importantes según esos indicadores.

Se debe agregar a lo anterior que cinco demandas (que se han subrayado) incluyen algún elemento que exige que el docente sea capaz de encontrar un justo medio en su comportamiento (o sepa moverse en una "delgada línea"): introducir su experiencia personal en los cursos, pero no "divagar" sobre su biografía; generar participación, pero no abdicar de la conducción de la docencia; generar relaciones no autoritarias, pero mantener el dominio del grupo curso; exigir rendimiento a los estudiantes, pero no sobrecargarlos; planificar y desarrollar todos los contenidos del programa, pero ser capaz de adaptarlo a las motivaciones emergentes de los estudiantes.

Figura 1. Demandas estudiantiles a la Docencia en la FACSO

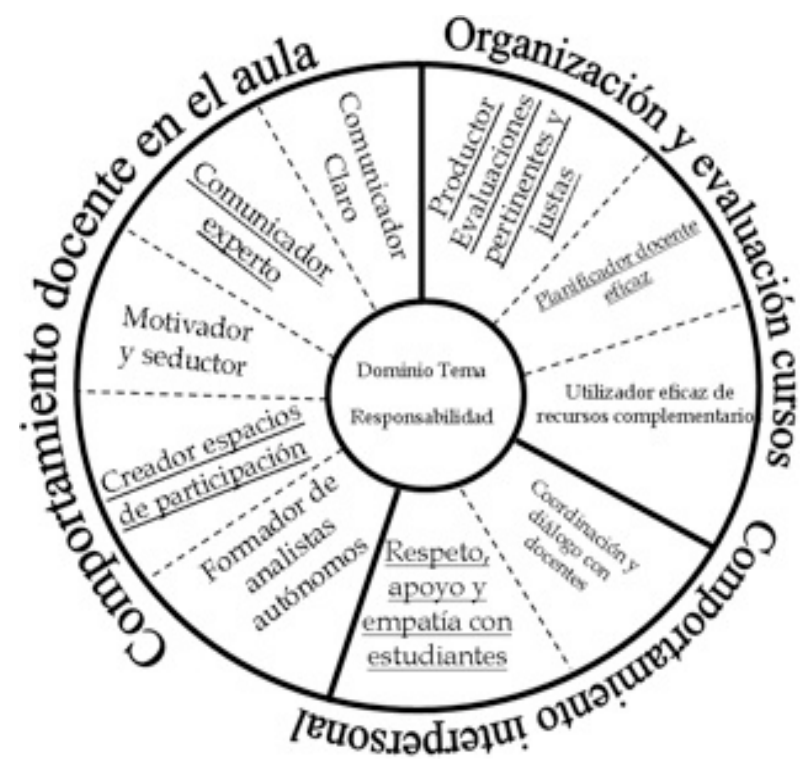

En relación a ello, se puede considerar también que los cursos son internamente heterogéneos y, por lo mismo, aunque la demanda sea nominalmente igual, es factible pensar que habrá distintos grupos de estudiantes que sitúen la línea óptima en lugares distintos.

Se suma a lo dicho, el hecho de que la demanda mayoritaria de estos estudiantes privilegia una formación reflexiva y centrada en la comprensión de los elementos que les son cercanos (biográfica o coyunturalmente), lo que puede implicar un fuerte desafío, tanto para los académicos que crean en la virtud de la entrega de herramientas operativas y se resistan a interpretarlas epistemológica o ideológicamente, como para aquellos que tengan una mirada más centrada en lo disciplinario y por ello sean reacios a hacer demasiado circunstancial su docencia.

También se debe tener en cuenta que los estudiantes se sitúan en una posición ambigua en relación con el conocimiento: si bien por un lado reconocen que no son expertos 
(y en ese sentido están en una posición subordinada a sus profesores), por otro lado -y especialmente en los cursos superiores-, los alumnos parecen considerar que ya están en condiciones de participar del debate intelectual. Piden tener "voz" a través de sus trabajos, pruebas u opiniones en clases. Por ello, son muy sensibles, tanto a lo que perciben como intentos de disminuir la importancia formativa del pregrado frente al postgrado, como a que los docentes hagan muy patente la diferencia que tienen con los estudiantes.

Además, se debe incorporar a la descripción que esta demanda no sólo es múltiple, difícil de graduar y compleja, sino también interconectada, pues, como se ha visto en el relato de cada una de las peticiones, la mayor parte de ellas requiere en cierta medida de otras para materializarse. Así, por ejemplo, para ser un buen productor de espacios de participación, se requiere generar relaciones de respeto con los estudiantes o para ser un comunicador claro, se debe planificar bien la organización del curso.

El problema para los académicos no es sólo que estén sometidos a expectativas altas, sino que también algunos alumnos tengan la impresión de que lo que están solicitando es simple. A ello se debe agregar que una de las matrices de explicación de la mala docencia que manejan los estudiantes, es la falta de interés de los académicos en su labor docente, su "falta de voluntad".

La relativa complejidad de la demanda estudiantil se contrapone con una estructura Universitaria que usualmente no ha asignado a la docencia de pregrado demasiada importancia en los criterios de contratación, calificaciones o carrera académica de sus integrantes, priorizando especialmente las labores de investigación, pero que de igual manera exige a todos sus integrantes realizar similares esfuerzos en la enseñanza. En ese contexto, no resulta insensato esperar que el nivel general de experticia y cercanía con las destrezas pedagógicas de los académicos sea muy irregular.

Así, si bien se dispone de algunos antecedentes que señalan que, al menos en el discurso explícito, los profesores reconocen las complejas demandas de los estudiantes actuales (Solar y Díaz, 2009), en el sentido de entender que se solicita un profesor motivador, entusiasta, cercano y preocupado por sus estudiantes, la información existente actualmente parece indicar que esa comprensión es sólo parcial y podría no estar completamente difundida o no traspasarse a las prácticas docentes concretas.

¿Qué se puede hacer en este escenario? Evidentemente, el desafío para las Universidades Chilenas consiste en transformar y mejorar la calidad de la docencia de pregrado, optimizando al mismo tiempo sus desempeños en el campo de la investigación y extensión. Posiblemente, ello exija acciones tanto técnicas como políticas. En el plano técnico el reto para las instituciones de educación superior puede consistir en reconocer la complejidad y relevancia que tiene en la actualidad la formación de pregrado en las labores de una Universidad, lo que se puede materializar en incorporar las habilidades docentes en los criterios de ingreso y desarrollo de la carrera académica; generar recorridos profesionales que permitan concentrarse en aquello que hacen mejor los académicos desigualmente dotados para la docencia o la investigación; instalar y legitimar un sistema de observación, seguimiento y apoyo a la docencia, facilitando a los profesores obtener capacitación en las habilidades que tengan deficitarias; y socializar en la totalidad de los académicos las nuevas demandas de los estudiantes, permitiendo una discusión y priorización informada de las acciones a seguir, entre otras posibles alternativas.

Desde el punto de vista político, se debe aceptar que si el objetivo es mejorar la calidad de la docencia sin comprometer las labores de investigación y extensión que 
actualmente realizan las instituciones de educación superior, se requiere invertir importantes recursos e iniciar nuevos debates que instauren un nuevo proyecto país para la educación superior, pues es claro que no será fácil para los académicos y las Universidades responder a la compleja demanda de sus alumnos y de la sociedad, en las condiciones institucionales actuales.

De esta manera, mejorar los niveles de aprendizaje de los estudiantes implica pensar nuevamente la forma en que se entiende la calidad docente, el nivel de conocimientos y destrezas que deben tener los egresados de la educación superior y el tipo de estructura institucional y pedagógica necesaria para formar esas competencias, al mismo tiempo que se continúa contribuyendo a la generación y difusión del conocimiento. Todo esto conduce necesariamente a establecer una discusión sobre las funciones y financiamiento de las Universidades y la manera de potenciar su contribución al progreso de Chile.

La promesa de iniciar estos debates y, eventualmente, establecer estos nuevos acuerdos es no sólo aumentar el nivel de satisfacción de los estudiantes con la educación que han recibido, sino también mejorar su contribución y la de las instituciones de educación superior al desarrollo nacional.

Finalmente, en relación con futuros estudios que motivan los resultados de la presente investigación, se pueden señalar tres: i) indagar el grado de generalización de los hallazgos acá presentados a estudiantes de otras facultades y Universidades chilenas; ii) explorar el discurso que los profesores tienen sobre los estudiantes y sus demandas; iii) describir la percepción que tienen los académicos sobre su enseñanza, sus gratificaciones, frustraciones y la importancia que le asignan en el contexto del resto de sus actividades profesionales. Es posible pensar que disponer de esa información permitirá comprender mejor la compleja dinámica de la docencia en el actual sistema Universitario chileno.

\section{REFERENCIAS BIBLIOGRÁFICAS}

Aguilar, A., Ampuero, N. y Loncomilla, L. (2007). Evaluación del desempeño docente: diagnóstico desde la perspectiva de los estudiantes. En CINDA-MINEDUC, Evaluación del desempeño docente y calidad de la docencia universitaria (pp. 71-79). Santiago de Chile: CINDA - MINEDUC.

Aleamoni, L. (1999). Student rating myths versus research facts from 1924 to 1998. Journal of Personnel Evaluation in Education, vol. 13, n. 2, 153-166.

Báez, M., Cazenave, M. y Lagos, J. (2007). Percepción de la evaluación del desempeño docente: diagnóstico desde la perspectiva del docente. En CINDA-MINEDUC, Evaluación del desempeño docente y calidad de la docencia universitaria (pp. 47-79). Santiago de Chile: CINDA - MINEDUC.

Chonko, L, Janner, J. \& Davies, R. (2002). What are thinking? Students` expectations and self assessments. Journal of Educational for Business, vol. 77, n. 5, 271-281.

Contreras, G. (2010). Diseño y operación de un sistema de evaluación del desempeño docente con fines formativos: la experiencia de la Pontificia Universidad Católica de Valparaíso, Chile. Revista Iberoamericana de Evaluación Educativa, vol. 3, n. 1, 180-191.

Contreras, G., Faúndez, F., Gutiérrez, A., Jiménez, G. Ponce, M. y Silva, E. (2007). Diagnóstico del proceso e instrumentos usados actualmente para la evaluación del desempeño docente. En CINDA-MINEDUC, Evaluación del desempeño docente y calidad de la docencia universitaria (pp. 81-98). Santiago de Chile: CINDA - MINEDUC. 
De La Fuente, H., Marzo, M. y Reyes, M.J. (2010). Análisis de la satisfacción universitaria en la facultad de ingeniería de la Universidad de Talca. Revista Chilena de Ingeniería, vol. $18, n$. $3,350-363$.

Flick, U. (2002). An introduction to qualitative research. London: SAGE.

Francis, S. (2006). Hacia una caracterización del docente universitario "excelente": una revisión a los aportes de la investigación sobre el desempeño del docente universitario. Revista Educación, vol. 30, n. 1, 31-39.

Gallardo, G. Y Reyes, P. (2010). Relación profesor - alumno: arista fundamental para el aprendizaje. Calidad de la Educación, vol. 32, 78-108.

Glaser, B. \& Strauss, A. (1967). The discovery of grounded theory. Chicago: Aldine.

Greimel-Fuhrmann, B. \& Geyer, A. (2003). Students' evaluation of teachers and instructional quality - analysis of relevant factors based on empirical research. Assessment and Evaluation in Higher Education, vol. 28, n. 3, 229-238.

Marín, M. y Teruel, M. (2004). La formación del docente universitario: necesidades y demandas desde su alumnado. Revista Universitaria de Formación del Profesorado, vol. 18, n. 2, 131-151.

Marsh, H. (1991). Multidimensional students evaluations of teaching effectiveness: a test of alternative higher - order structures. Journal of Educational Psychology, vol. 83, n. 2, 285-296.

Morgan, D.L. (1990). Focus groups as qualitative research. Newbury Park: Sage.

Naser-Abu, F. \& Frasko, B. (2009). Student evaluation of instruction: what can be learned from students' written comments. Studies in Educational Evaluation, vol. 35, 37-44.

Jahangiri, L. \& Mucciolo, T. (2008). Characteristics of effective classroom teachers as identified by students and professionals: a qualitative study. Journal of Dental Education, vol. 72, $n$. 4, 707-718.

Okpala, C. \& Ellis, R. (2005). The perceptions of college students on teacher quality: a focus on teacher qualifications. Education, vol. 126, n. 2, 374-383.

Pozo, C., Rebolloso, E. \& Fernández, B. (2000). The "ideal teacher". Implications for student evaluation of teacher effectiveness. Assessment and Evaluation in Higher Education, vol. 25, n. 3, 253-263.

Salazar, J. (2010). Encuesta de satisfacción estudiantil versus cultura evaluativa de la docencia. Revista Iberoamericana de Evaluación Educativa, vol. 3, n. 1e, 121-132.

Salazar, J. (2008). Diagnóstico preliminar sobre evaluación de la docencia universitaria. Una aproximación a la realidad en las universidades públicas y/o estatales de Chile. Revista Iberoamericana de Evaluación Educativa, vol. 1, n. 3, 67-84.

Solar, M. y Díaz, C. (2009). El profesor universitario: Construcción de su saber pedagógico e identidad profesional a partir de sus cogniciones y creencias. Calidad en la Educación, vol. 30, 208-232.

Strauss, A. \& Corbin, J. (1990). Basics of qualitative research. Newbury Park: Sage.

Villalobos, A., Melo, Y. y Pérez, C. (2010). Percepción y expectativas de los alumnos universitarios frente al profesor no pedagogo. Estudios Pedagógicos, vol. 36, n. 2, $261-269$.

Zúñiga, M. y Jopia, B. (2007). La evaluación del desempeño docente en las universidades chilenas: Diagnóstico desde la perspectiva de las autoridades universitarias. En CINDA-MINEDUC. Evaluación del desempeño docente y calidad de la docencia universitaria (pp. 30-46). Santiago de Chile: CINDA - MINEDUC. 